\title{
Evaluación en el nivel de conocimiento de las madres después de aplicada una estrategia educativa. Venezuela 2015
}

\section{Evaluation of level of knowledge among mothers after applying an educational strategy. Venezuela 2015}

\section{RESUMEN}

La lactancia materna es un acto natural de alimentación, pero al mismo tiempo es un comportamiento aprendido. El objetivo fue evaluar el nivel de conocimiento de las madres y embarazadas después de aplicada una estrategia educativa. Venezuela, 2015. Se realizó una investigación de tipo evaluativa, de corte transversal en 1.132 embarazadas y madres de niños menores de dos años, aparentemente sanas. Se midió el grado de conocimiento antes de la intervención a través de un pre-test. Posteriormente, se realizaron talleres educativos en temas de lactancia materna: beneficios, fisiología, aportes nutricionales de la leche materna, conservación y almacenamiento de la leche materna, técnicas de amamantamiento, leyes que protegen la práctica y se aplicó nuevamente el test. Para el procesamiento y análisis de la información se utilizó el programa estadístico SPSS 19.0. Entre los resultados están el aumento del nivel de conocimiento de $28,8 \%$ (deficiente) a $60,8 \%$ (bueno), observándose una correlación significativa $(p<0,05)$ en el número de respuestas correctas antes y después del test. Los temas que tuvieron un mayor impacto fueron: conservación de la leche, práctica de amamantamiento y medidas para incrementar la producción de leche. Se concluye que las madres y embarazadas evaluadas mejoraron sus conocimientos en temas de lactancia materna debido en parte a la estrategia educativa emprendida.

Palabras clave: Conocimiento; Educación en lactancia; Embarazadas; Lactancia materna; Maternidad.

\footnotetext{
ABSTRACT

Breastfeeding is a natural feeding act, but, at the same time, it is a learned behavior. The objective of this study was to evaluate the level of knowledge of mothers and pregnant women after applying an educational strategy in Venezuela. An evaluative, cross-sectional investigation was conducted in 2015 among 1.132 pregnant women and mothers of children under two years of age who were apparently healthy. The degree of knowledge before the intervention was measured through a pre-test. Subsequently, educational workshops were held on breastfeeding topics: benefits, physiology, nutritional contributions of breast milk, conservation and
}

Jaclyn Rojas" , Ivo Contreras² , Crismari Chaparro', Ángela Quintero², Roshman González³.

1. Instituto Nacional de Nutrición. Caracas, Venezuela. 2. Universidad de los Andes. Santiago, Chile. 3. Universidad Central de Venezuela.

\begin{abstract}
Dirigir correspondencia a: Jaclyn Rojas. Instituto Nacional de Nutrición. Avenida Baralt, Esquina El Carmen, Edificio INN, Municipio Libertador, Caracas, Venezuela. Teléfono: +584122490125 . Correo electrónico: jaclynrojas@gmail.com
\end{abstract}

Este trabajo fue recibido el 12 de junio de 2017. Aceptado con modificaciones: 04 de julio de 2018. Aceptado para ser publicado: 16 de octubre de 2018.

storage of breast milk, breastfeeding techniques, laws that protect the practice, after which, the test was applied again. For the processing and analysis of the information, the statistical program SPSS 19.0 was used. The knowledge level increased from $28.8 \%$ (deficient) to $60.8 \%$ (good). We also observed a significant correlation $(p<0.05)$ in the number of correct answers before and after the test. The topics that had the greatest impact were: milk conservation, breastfeeding practice and measures to increase milk production. In conclusion, mothers and pregnant women improved their knowledge of breastfeeding issues due in part to the impact of the educational activities undertaken. Key words: Breastfeeding; Breastfeeding Education; Knowledge; Maternity; Pregnant women. 


\section{INTRODUCCIÓN}

La lactancia materna (LM) es vital para el comienzo óptimo de la vida puesto que proporciona el alimento ideal durante los primeros seis meses de vida del niño, además de aportar todos los nutrientes, anticuerpos, hormonas, factores inmunitarios y antioxidantes que se necesita para un desarrollo sano.

Actualmente existe un renovado interés por rescatar la cultura del amamantamiento, basado en los beneficios que aporta esta práctica de alimentación, dado que proporciona todas las sustancias y nutrientes que los infantes necesitan para su desarrollo físico y mental, así como por la protección que ofrece contra enfermedades frecuentes durante esta etapa de la vida, todo ello contribuyendo a disminuir la morbilidad y mortalidad infantil. Asimismo, se sostiene que esta práctica también beneficia a las madres al reducir el riesgo de cáncer de mama y de ovarios, además de su efecto en el espaciamiento de los embarazos y en los niveles de fecundidad.

Un número cada vez mayor de estudios han demostrado que la LM tiene, tanto a corto como a largo plazo, efectos beneficiosos sobre el sistema cardiovascular materno, actuando sobre los factores de riesgo, tales como el nivel de lípidos, la obesidad, la presión arterial, la insulina y los niveles de glucosa Las mujeres que dan el pecho a sus hijos presentan parámetros metabólicos más favorables y recuperan su peso anterior al embarazo de forma más rápida. Por otro lado, diversos estudios han demostrado que esas mujeres presentan menos riesgos de padecer el síndrome metabólico, enfermedades cardiovasculares y cáncer de mama en el futuro. Así pues, la LM se debe promover tanto para la salud del bebé como de la madre ${ }^{1,2}$.

Es preocupante cómo se ha modificado su práctica de forma negativa, influida por factores sociales, económicos, culturales y psicológicos. Sin embargo, el "entorno social y familiar de la madre es trascendental para el éxito de la lactancia materna", ya que es una influencia cultural y una fuente de apoyo potencial para la toma de decisiones con respecto a la forma de alimentación que ella considerará como adecuada para su hijo ${ }^{3}$, y donde las tensiones entre los conocimientos populares de las madres y los conocimientos científico - disciplinares de los agentes institucionales ${ }^{4}$ generan mayores controversias.

Diversos autores confirman que la forma en que una madre cuida de su hijo, depende de una mezcla compleja de su constitución genética, la respuesta del niño, una larga historia de relaciones interpersonales, su entorno familiar, orientación médica, factores sociales y culturales, entre otros; de allí que el aprendizaje tiene un papel fundamental en la lactancia materna, pero es un aprendizaje centrado en las creencias, actitudes y valores de la cultura ${ }^{4,5,6}$.

El objetivo principal de esta investigación es evaluar el nivel de conocimiento de las madres y embarazadas después de aplicada una estrategia educativa.

\section{MATERIALES Y MÉTODOS}

Se realizó una investigación evaluativa, de corte transversal en embarazadas y madres de niños menores de dos años, de 18 estados del país durante el periodo octubre-diciembre del 2015.

El muestreo fue no probabilístico por conveniencia, quedando constituido por 1.132 embarazadas y madres residentes de la zona por más de 1 año. Fueron excluidas aquellas embarazadas y madres menores de 12 años y que no firmaron el consentimiento informado.

Se construyó un instrumento para medir el conocimiento en temas de lactancia a las madres y embarazadas, que fue validado a través de una prueba piloto en 25 participantes. Posteriormente, se aplicó la prueba a las madres y embarazadas. La evaluación constó de 12 preguntas con cinco opciones de respuestas y en las cuales solo una era la correcta, posteriormente se asignó la puntuación de 1 por cada ítem correcto y 0 por cada ítem con respuesta errónea de acuerdo al número de elementos respondidos correctamente, se categorizó el nivel de conocimiento de la siguiente manera: 0-3 "deficiente", 4-6 "regular", 7-9 "bueno" y de 10-12 "excelente".

Se realizaron sesiones educativas en temas de lactancia materna: beneficios, fisiología, aportes nutricionales de la leche materna, conservación y almacenamiento de la leche materna, técnicas de amamantamiento, leyes que protegen la práctica; durante dos sesiones de 60 minutos cada una y con el empleo de diferentes formas de organización tales como: conferencia, conversatorio, medios audiovisuales y dinámicas que permitieran el análisis y la reflexión. Una vez concluida la intervención, se aplicó nuevamente la prueba de conocimiento (Anexo 1).

Adicionalmente se clasificó como efectiva la estrategia educativa si el $50 \%$ o más de las participantes habían adquirido un nivel de conocimiento superior al obtenido anteriormente en el pre-test.

Se realizó la validación de la prueba a través de juicio de expertos correspondientes a las áreas de nutrición, educación y sociología del personal técnico del Instituto Nacional de Nutrición, adicionalmente se realizó una estandarización a los coordinadores estadales y responsables de la investigación (nutricionista y educadores); como producto final de esta sesión se elaboró un documento resumen con las directrices metodológicas.

\section{Análisis Estadístico}

Para el procesamiento y análisis de la información se utilizó el programa estadístico SPSS versión 19.0. Se realizaron estadísticas descriptivas (frecuencias absolutas y relativas) y correlaciones entre variables (Chi cuadrado).

Para una mejor presentación de la información se clasificó la edad de las madres en seis categorías: adolescente temprana (<13 años), adolescente media (14-16 años), adolescente tardía (17-19 años), adulta joven (20-35 años) y adulta tardía (36 años y más). 


\section{Consideraciones Éticas}

Previo al llenado del cuestionario se realizó por escrito el consentimiento informado para su participación confidencial y voluntaria en la investigación quedando reflejado por escrito los principios fundamentales de la bioética como son la responsabilidad, justicia, autonomía, precaución; destacando los derechos y deberes de los participantes y los investigadores en el estudio según lo establecido por el Código de Ética para la Vida del Ministerio del Poder Popular para la Ciencia, Tecnología e Industrias Intermedias.

\section{RESULTADOS}

En primer lugar, se refleja las características sociodemográficas de las madres y embarazadas pertenecientes a zonas urbanas $(47.6 \%)$ seguidamente de un $36.6 \%$ de zona rural. En cuanto al nivel educativo de la muestra seleccionada en su mayoría eran bachiller $33.2 \%$ y un $22.9 \%$ corresponden a secundaria incompleta. Al indagar en la condición laboral tenemos que el $67 \%$ trabaja y un 33,0\% no realiza ninguna actividad laboral (Tabla 1 ).

Tabla 1. Características Socio-demográficas de las madres.

\begin{tabular}{|c|c|c|c|}
\hline \multicolumn{2}{|c|}{ Características Sociodemográficas } & \multirow{2}{*}{$\frac{\mathbf{N}^{\mathbf{o}}}{539}$} & \multirow{2}{*}{$\begin{array}{c}\% \\
47,6\end{array}$} \\
\hline Procedencia & Urbana & & \\
\hline & Rural & 448 & 39,6 \\
\hline & Indígena & 145 & 12,8 \\
\hline \multirow[t]{8}{*}{ Nivel educativo } & No lee ni escribe & 55 & 4,9 \\
\hline & Primaria incompleta & 76 & 6,7 \\
\hline & Primaria completa & 74 & 6,5 \\
\hline & Secundaria incompleta & 259 & 22,9 \\
\hline & Bachiller & 376 & 33,2 \\
\hline & Universitaria incompleta & 149 & 13,1 \\
\hline & Universitaria completa & 137 & 12,1 \\
\hline & Postgrado & 6 & 0,6 \\
\hline \multirow[t]{3}{*}{ Condición laboral } & Trabaja & 763 & 67,0 \\
\hline & No trabaja & 369 & 33,0 \\
\hline & Total* $^{*}$ & 1132 & 100,0 \\
\hline
\end{tabular}

Adicionalmente se evidenció que el $48 \%$ correspondió a embarazadas y el $52 \%$ a madres de niños menores de 24 meses. El grupo de edad que prevaleció fue de 20 a 35 años, lo cual representó el $66 \%$ de la muestra evaluada; siendo la edad promedio de 24 años $\pm 6,5$, seguido del grupo de adolescente tardía (19\%) (Tabla 2).

Al realizar la prueba de evaluación de conocimiento (pre test) en temas vinculados a la lactancia materna, se evidenció un desconocimiento general en contenidos como: beneficios de lactancia, duración, técnicas, conservación y almacenamiento de la leche materna, leyes que amparan la práctica de la lactancia, entre otros, observándose un alto porcentaje de respuestas incorrectas que clasifican a las madres evaluadas con un nivel de conocimiento deficiente (65\%).

Posteriormente se realizaron talleres educativos en tópicos como: los conceptos básicos, la fisiología, la importancia y beneficios de la lactancia materna, técnicas para amamantar, métodos de extracción y conservación de la leche materna y marco legal. Al realizar la prueba de conocimiento después de la intervención educativa (pos test), se observó un incremento en el porcentaje de respuestas correctas para los temas antes mencionados, incrementándose el nivel de conocimiento en la categoría "bueno" y "excelente", situación que no se evidenció en la evaluación de conocimiento inicial (Figura 1). A través de la prueba chi-cuadrado con una significación estadística de 0.05 se pudo demostrar la efectividad de las estrategias educativas impartida. Es importante destacar que las preguntas contentivas a los temas de conservación y almacenamiento de leche materna producción de leche y técnicas de amamantamiento; las madres y embarazadas entrevistadas aumentaron significativamente su conocimiento (Figura 2).

\section{DISCUSIÓN}

La Organización Mundial de la Salud (OMS) en su 55 Asamblea Mundial refiere que una práctica adecuada de la lactancia materna es fundamental para la supervivencia, el crecimiento, desarrollo de la salud y la nutrición de los lactantes y niños en cualquier lugar del mundo?. Sin embargo, menos del $40 \%$ de las madres alrededor del mundo amamantan a sus hijos exclusivamente durante los primeros seis meses de vida ${ }^{8}$.

Tabla 2. Grupos de edad de las participantes según condición. Venezuela 2015. Nota: El 7,7\% de madres y embarazadas NR/NC.

\begin{tabular}{|c|c|c|c|c|c|c|}
\hline & \multicolumn{2}{|c|}{ Embarazadas } & \multicolumn{2}{|c|}{ Madres } & \multicolumn{2}{|c|}{ Total } \\
\hline Adolescente media & 52 & 11,13 & 37 & 6,41 & 89 & 8,52 \\
\hline Adolescente Tardía & 105 & 22,48 & 97 & 16,81 & 202 & 19,35 \\
\hline Adulta joven & 281 & 60,17 & 409 & 70,88 & 690 & 66,09 \\
\hline Total & 467 & 100 & 577 & 100 & 1.044 & 100 \\
\hline
\end{tabular}




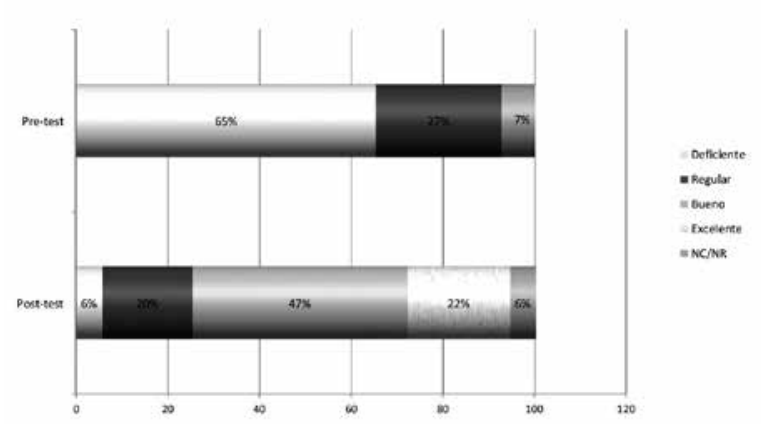

Figura 1. Nivel de conocimientos en temas de lactancia materna en madres y embarazadas. Venezuela 2015.

En este sentido, la lactancia está determinada por un cúmulo de saberes de las madres, los cuales se describen como el conjunto de conceptos y creencias que ellas tienen sobre la lactancia. Estos son el producto del intercambio de mensajes a través de generaciones, de las culturas y costumbres de las comunidades; y a ellos se suman los conceptos impartidos por los agentes de salud. Toda ésta información de carácter técnico, científico o del saber popular, es organizada y apropiada de manera racional por la madre y dentro de su lógica explica y justifica los comportamientos frente a la práctica de la lactancia. Actualmente se sabe que la educación en lactancia materna durante la atención prenatal y/o posnatal aumenta la incidencia de la misma 9 .

En este estudio, el grupo de edad de las madres evaluadas estuvo comprendido entre 13 y 48 años, datos coincidentes con los de Gorrita y cols..$^{10}$ que reporta un mayor predominio en el grupo de edad entre 20 y 34 años $(88,4 \%)$, siendo esta la etapa óptima para la concepción, pues la mujer ya ha completado su maduración biológica y está mejor preparada para la procreación.

Son múltiples las investigaciones en que se ha analizado la relación entre la duración de la lactancia materna y la edad de la madre; algunas, como la realizada por López A y cols. ${ }^{11}$, señalan que a medida que aumenta el nivel de escolaridad materna se incrementa el número de madres que lactan a sus hijos durante más de un mes.

Difiere de estos resultados Avalos $\mathrm{M}^{4}$, al plantear que las madres con bajo nivel de escolaridad tienen los índices más altos de lactancia materna. En la actualidad existe un bajo porcentaje de madres adolescentes que lactan hasta el sexto mes y más ${ }^{12}$. Sin embargo, con procesos de enseñanzaaprendizaje y con métodos acordes se puede lograr un mayor conocimiento de estos temas tan importantes y propiciar el mantenimiento de una lactancia materna exclusiva hasta la primera mitad del primer año de vida.

Al realizar la intervención educativa en temas de lactancia materna se observó un progreso en el conocimiento adquirido por las madres y embarazadas participantes,

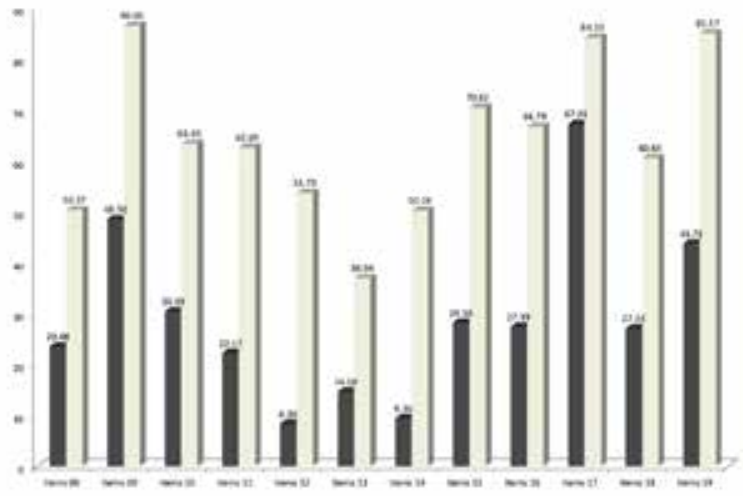

Figura 2. Conocimientos de madres y embarazadas por ítems evaluado. Venezuela 2015.

mostrándose un porcentaje de avance del $28,8 \%$ a $60,8 \%$, evidenciándose un incremento del más del $50 \%$ en el nivel de conocimiento de las madres y embarazadas en relación con su evaluación inicial (pre-test), evaluándose la estrategia educativa como efectiva para este grupo de participantes. López y $\mathrm{Col}^{14}$ en su estudio pone de manifiesto la efectividad de una intervención educativa en lactancia materna en donde inicialmente 29 madres tenían un conocimiento evaluado de inadecuado (80.55\%), y al final de la intervención educativa sólo dos madres (5.55\%) tenían esta evaluación.

Bocanegra, Espinoza y Ruiz' ${ }^{15}$, muestran la efectividad de una intervención educativa, al evaluar el nivel de conocimiento sobre lactancia materna exclusiva en las primigestas adolescentes obteniendo como resultado en el pre test, que un $19 \%$ presentaron un nivel de conocimiento alto, mientras que un $33 \%$ presento un nivel de conocimiento bajo. Luego de la aplicación del post test, el $48 \%$ presento un nivel de conocimiento alto y un $14 \%$ presento un nivel de conocimiento bajo. Datos coincidentes con los reportados en esta investigación que muestran la efectividad de la estrategia educativa impartida.

Por otra parte, al indagar en las preguntas contentivas a la "conservación y almacenamiento de la leche materna", "aumento en la producción de leche", y "técnicas de amamantamiento", las madres y embarazadas aumentaron su nivel de conocimiento. Datos coincidentes con los reportados por Rivadeneyra y Huyhua ${ }^{16}$, en donde se evidencio que del $100 \%$ (40) de las madres encuestadas; $82,5 \%$ (33) percibió un nivel de conocimiento medio y el $17,5 \%$ (7) nivel bajo. Según las dimensiones: importancia de LME el 75\% (30) percibió un nivel de conocimiento bajo y $25 \%$ (10) nivel medio, en la dimensión beneficios de LME el $65 \%$ (26) percibió un nivel de conocimiento medio y el $35 \%$ (14) nivel bajo, en ventajas de LME el 65\% (26) percibió un nivel de conocimiento bajo, y $35 \%$ (14) nivel medio, en la dimensión técnicas de LME el 85\% (34) percibió un nivel de conocimiento medio y el $15 \%(6)$ nivel bajo ${ }^{16}$. 
De las muchas resoluciones, iniciativas, políticas y directrices mundiales aún pertinentes que tienen como propósito proteger, promover y apoyar la lactancia materna, son necesarias nuevas estrategias de promoción, sustentadas con datos científicos. Estas nuevas estrategias deben estar fundamentadas por los datos que confirman que los niños amamantados quizá estén mejor preparados para la economía mundial dadas las consecuencias positivas sobre los niveles de inteligencia ${ }^{17}$; que las madres quizá estén protegidas de enfermedades como el cáncer; la familia y la sociedad se beneficiarán gracias al ahorro en servicios de atención de salud y que se protegería el ambiente de los millones de latas y biberones que hoy son desechados en los vertederos. Es importante promover un ambiente social en el cual las madres se sientan libres para amamantar donde sea y cuando sea.

Anexo 1. Preguntas del pre test y pos test.

INTERCAMBIO DE SABERES CON PRENATALES Y MADRES LACTANTES (Zonas urbanas, rurales e indigenas)

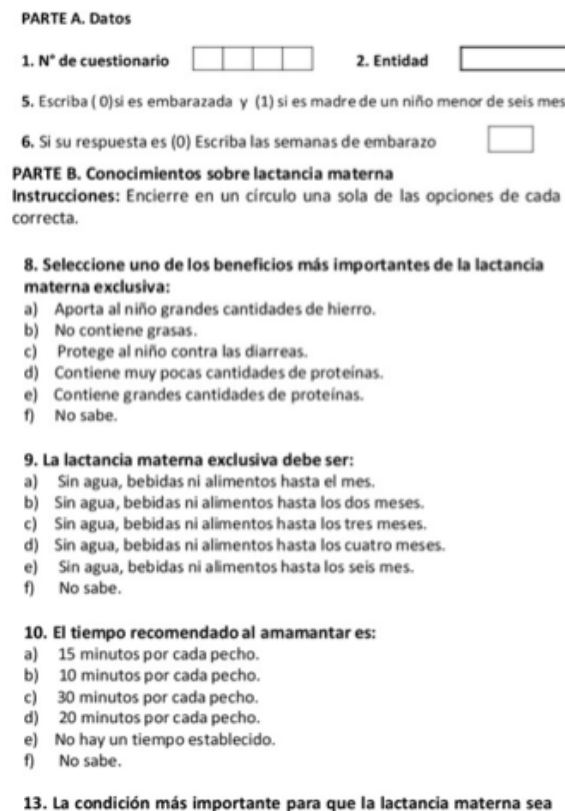

13. La condición más importante para que la lactancia materna sea exitosa es que:

a) La madre tome abundantes liquidos para aumentar la producción. b) La madre mantenga una actitud positiva al amamantar.

c) La madre tenga los senos grandes de modo que pueda producir abundante leche.

d) La madre esté bien alimentada.

e) La madre posicione bien al niño para que tenga un agarre correcto del pecho.

f) No sabe.

14. Para estimular que la leche fluya en los pechos, es necesario:

a) Consumir bebidas a base de leche de vaca.

b) Que la madre reciba masajes en la base de la cabeza.

c) Utilizar bebidas caseras.

d) No estimular los pezones para evitar lastimarlos o producir dolor.

e) Solicitarle a su médico que le indique medicamentos.

f) No sabe.

15. Para que la mamada sea buena y el niño quede satisfecho, se debe observar lo siguiente:

a) Las mamadas deben ser rápidas.

L) Los labios del niño deben estar en forma de boca de pescado.

c) El niño debe agarrar solo los pezones con los labios para no maltratar las mamas.

d) El labio inferior del niño debe agarrar solo el pezón.

e) La barbilla no debe tocar el pecho de la mama.

No sabe.
3. Edad de la Madre:
4. Participante $N^{*}$;

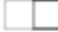

eses

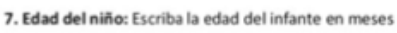

7. Edad del niño: Escriba la edad del infante en meses

7. Edad del niño: Escriba la edad del infante en meses
$\square$

11. El calostro es conocido como:

a) La leche mala o podrida. b) Es un alimento rico en grasas para reducir la resequedad de

c) Es un alimento rico en agua y fibra para ayudar a eliminar los restos de anestesia al momento del nacimiento.

d) La leche de los primeros dias que favorece la expulsión de las primeras heces del niño $y$ ayuda a prevenir que se ponga amarillo.

e) Es la leche que contiene grandes cantidades de hierro para aumentar las defensas del niño.

f) No sabe.

12. El tiempo recomendado para la conservación de la leche materna luego de haberse ordeñado es:

a) Hasta dos horas fuera de la nevera.

b) Hasta tres horas fuera de la nevera.

c) Hasta seis horas fuera de la nevera.

c) Hasta ochohoras fuera de la nevera.

d) Hasta ocho horas fuera de la nevera.

f) No sabe.

16. Cuando no se ha creado un centro de educación inicial en el sitio de trabajo, las mujeres que amamantan tienen derecho a:

a) Dos descansos diariamente de una hora y media cada uno.

b) Dos descansos diariamente de una hora cada uno.

c) No tiene derecho a descansos durante la jornada laboral.

d) Solo tiene derecho a descansos, la madre que tiene hijos en condiciones de discapacidad.

e) Un descanso media hora cada uno diariamente.

f) No sabe.

17. Luego de parir, el recién nacido hospitalizado tiene derecho de estar junto a su madre:

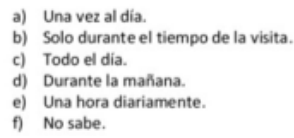

18. La embarazada trabajadora tiene derecho a:

a) Un descanso de seis semanas antes del parto y 12 semanas después.

a) Un descanso de seis semanas antes del parto y 12 semanas después. despues.

c) No tiene derecho a descanso después del parto.

d) No tiene derecho a descanso antes del parto.

e) Un descanso durante seis semanas antes del parto y 20 semanas después.

f) No sabe.

19. Los padres trabajadores con hijos recién nacidos, tienen derecho a un permiso de:

a) Siete dias continuos.

b) Tres dias continuos.

c) No tiene derecho a permiso.

d) 14 catorce dias continuos.

e) Nueve dias continuos.

f) No sabe. 


\section{CONCLUSIONES}

La estrategia educativa en lactancia materna resultó ser efectiva en las madres y embarazadas participantes del estudio ya que se evidenció un incremento en el número de respuestas correctas después de aplicada la prueba de conocimiento. Los temas en los cuales se observó un notorio incremento del conocimiento fueron: "conservación de la leche", "práctica de amamantamiento" y "medidas para incrementar la producción de leche".

\section{BIBLIOGRAFÍA}

1. Aguilar M, Sánchez A, Madrid N, Mur N, Expósito M, Hermoso E. Lactancia materna como prevención del sobrepeso y la obesidad en el niño y el adolescente: revisión sistemática. Nutr. Hosp. [Internet]. 2015 Feb [citado 2018 Jul 11]; 31(2): 606-620. Disponible en: http://scielo.isciii.es/scielo. php?script=sci_arttext\&pid=S0212-16112015000200010\&/ng=es. http://dx.doi.org/10.3305/nh.2015.31.2.8458.

2. Aguilar $M$, García L, Sánchez A, Barrilao R, Rodríguez $E$, Villar M. Beneficios inmunológicos de la leche humana para la madre y el niño. Revisión Sistemática. Nutr Hosp 2016; 33(2): 482-493.

3. Bermudez F, Rocha L, Fonseca D, Bermudez L. El entorno familiar y social de la madre como factor que promueve o dificulta la lactancia materna. Rev Fac Med Colombia 2015; 63(2): 217-227.

4. Avalos M; Mariño E; González J. Factores asociados con la lactancia materna exclusiva. Revista Cubana de Medicina General Integral 2016; 35(2).

5. Trujillo O, Peñaranda F, Otálvaro J, Bastidas M; Torres J. La lactancia materna como un fenómeno singular y sociocultural surcado por diferencias y tensiones. Archivos venezolanos de puericultura y pediatría 2015; 78(2): 45-51.

6. Quispe MP, Oyola AE, Navarro M, Silva JA. Characteristics and maternal beliefs associated to the cessation of exclusive breastfeeding [In Spanish]. Rev Cubana Salud Pública 2015; 41(4): 582-592.

7. OMS-UNICEF. Protection, Promotion and Support to Breastfeeding (Joint Declaration). Geneva: 55th Health Assembly 2002; 107.
8. World Health Organization. Indicators for assessing infant and young child feeding practices: conclusions of a consensus meeting held 6-8 November 2007 in Washington D.C. Geneva: World Health Organization; 2008.

9. Franco, José et al. Conocimiento sobre lactancia materna en embarazadas que acudieron a consulta prenatal: Hospital Dr. Patrocinino Peñuela Ruiz. San Cristóbal, Venezuela. Arch Venez Puer Ped [online] 2014; 77(3) [citado 2018-07-30], pp. 128-132. Disponible en: <http://www.scielo.org.ve/scielo. php?script=SCi_arttext\&pid $=$ S000406492014000300005\& $\mathrm{I}$ $n g=e s \& n r m=i s O>$. ISSN 0004-0649.

10. GorritaR, Britol, Ravelo Y, Ruiz E. Conocimientos de futuras madres sobre la lactancia materna y sus ventajas. Revista de ciencias médicas. La Habana. 2014; 20(1).

11. Annely Y. López Calzadilla, Escalona J, Gumucio M, Rodriguez $R$, Tejeda R. Estrategia de Intervención sobre Lactancia Materna. Gibara. 2014

12. Metas mundiales de nutrición 2025. Documento normativo sobre lactancia materna.

13. Gorrita R Terrazas Saldana A; Brito D, Ravelo Y. Some aspects related to exclusive breastfeeding in the first six months of life. Rev Cubana Pediatr 2015; 87(3): 285-297. http://scielo. sld.cu/scielo.php?script=sci_arttext\&pid=S00347531201500 0300004\&Ing=es\&nrm=iso. ISSN 1561-3119.

14. Guerra E, Valdes I, Martinez M, Aria Y, Martinez A. Conocimientos, actitudes, motivaciones y prácticas sobre lactancia materna vinculados a intervención educativa. Policlínico Jimmy Hirtzel. Bayamo- Granma. Multimed 2014; 18(2): 52-66.

15. Bocanegra L, Espinoza M, Ruiz A. Efectividad de una intervención educativa de enfermería sobre lactancia materna exclusiva en el conocimiento de primigestas adolescentes que acuden $i$ al centro de salud Laura Caller, Los Olivos, Perú 2016. [Tesis].

16. Rivadeneyra Z, Huyhua S. Nivel de conocimientos sobre lactancia materna exclusiva en madres que acuden al servicio de crecimiento y desarrollo, hospital regional Virgen de Fátima, Chachapoyas Amazonas- 2017. [Tesis].

17. Brahm $P$, Valdes $V$. Beneficios de la lactancia materna y riesgos de no amamantar. Rev Chil Pediatr 2017; 88(1): 07-14. https:// scielo.conicyt.cl/scielo.php?script=sci_arttext\&pid=S037041062 017000100001\&Ing=es\&nrm=iso >. ISSN0370-4106. http:// dx.doi.org/10.4067/S0370-41062017000100001. 